\title{
Epoxyeicosatrienoic Acids Regulate Adipocyte Differentiation of Mouse 3T3 Cells, Via PGC-1 $\alpha$ Activation, Which Is Required for HO-1 Expression and Increased Mitochondrial Function
}

\author{
Maayan Waldman, ${ }^{1,2,{ }^{*}}$ Lars Bellner, ${ }^{1, *}$ Luca Vanella, ${ }^{1,3,{ }^{\star}}$ Joseph Schragenheim, ${ }^{1}$ Komal Sodhi, ${ }^{4}$ \\ Shailendra P. Singh,, Daohong Lin,, Anand Lakhkar, Jiangwei Li, Edith Hochhauser, Michael Arad, \\ Zbigniew Darzynkiewicz, Attallah Kappas, and Nader G. Abraham ${ }^{1,4,7,8}$
}

Epoxyeicosatrienoic acid (EET) contributes to browning of white adipose stem cells to ameliorate obesity/ diabetes and insulin resistance. In the current study, we show that EET altered preadipocyte function, enhanced peroxisome proliferation-activated receptor $\gamma$ coactivator $\alpha(\mathrm{PGC}-1 \alpha)$ expression, and increased mitochondrial function in the 3T3-L1 preadipocyte subjected to adipogenesis. Cells treated with EET resulted in an increase, $P<0.05$, in PGC- $1 \alpha$ and a decrease in mitochondria-derived ROS (MitoSox), $P<0.05$. The EET increase in heme oxygenase-1 (HO-1) levels is dependent on activation of PGC- $1 \alpha$ as cells deficient in PGC- $1 \alpha$ (PGC- $1 \alpha$ knockout adipocyte cell) have an impaired ability to express HO-1, $P<0.02$. Additionally, adipocytes treated with EET exhibited an increase in mitochondrial superoxide dismutase (SOD) in a PGC-1 $\alpha$-dependent manner, $P<0.05$. The increase in PGC- $1 \alpha$ was associated with an increase in $\beta$-catenin, $P<0.05$, adiponectin expression, $P<0.05$, and lipid accumulation, $P<0.02$. EET decreased heme levels and mitochondria-derived ROS (MitoSox), $P<0.05$, compared to adipocytes that were untreated. EET also decreased mesoderm-specific transcript (MEST) mRNA and protein levels $(P<0.05)$. Adipocyte secretion of EET act in an autocrine/ paracrine manner to increase PGC- $1 \alpha$ is required for activation of HO-1 expression. This is the first study to dissect the mechanism by which the antiadipogenic and anti-inflammatory lipid, EET, induces the PGC-1 $\alpha$ signaling cascade and reprograms the adipocyte phenotype by regulating mitochondrial function and HO-1 expression, leading to an increase in healthy, that is, small, adipocytes and a decrease in adipocyte enlargement and terminal differentiation. This is manifested by an increase in mitochondrial function and an increase in the canonical Wnt signaling cascade during adipocyte proliferation and terminal differentiation.

\section{Introduction}

$\mathrm{T}$ HE CYTOCHROME P450 (CYP) monooxygenase/epoxygenase family is responsible for formation of 20-hydroxyeicosatetraenoic acid (20-HETE) and epoxyeicosatrienoic acids (EETs) [1,2]. Upon formation, EETs are rapidly hydrolyzed by soluble epoxide hydrolases (sEHs) and reactive oxygen species (ROS) [heme oxygenase-1 (preventable by HO-1) induction] to their respective dihydroxyepoxytrienoic acids (DHETs), as well as to esterification primarily to glycerophospholipids. Vasodilatory, anti-inflammatory, and antiapop- totic actions of EETs are well established, and sEH inhibition significantly increases cellular and circulating EET levels [3,4]. EET increases osteoblast differentiation but decreases adipocyte differentiation via activation of HO-1 (HO-1) [5-7]. In 3T3 adipocyte cells, an increase in heme increases adipogenesis $[8,9]$, which is associated with a glucose-mediated increase in ROS, which inactivates HO-1 (reviewed [10]).

The 3T3 adipocyte is a model to study the role of PGC-1 as thermogenic activators, the mitochondrial heme pathway, and biogenesis [11] as well as the evaluation of heme homeostasis [12]. However, an increase in HO-1 gene

Departments of ${ }^{1}$ Pharmacology, ${ }^{5}$ Pathology, and ${ }^{8}$ Medicine, New York Medical College, Valhalla, New York.

${ }^{2}$ Cardiac Research Laboratory, Felsenstein Medical Research Institute, Tel-Aviv University, Petah-Tikva, Israel.

${ }^{3}$ University of Catania, Department of Drug Science/Section of Biochemistry, Catania, Italy.

${ }^{4}$ Departments of Medicine and Surgery, Joan C. Edwards School of Medicine, Marshall University, Huntington, West Virginia.

${ }^{6}$ Leviev Heart Center, Sheba Medical Center, Tel Hashomer and Sackler School of Medicine, Tel Aviv University, Tel Hashomer, Israel.

${ }^{7}$ The Rockefeller University, New York, New York.

*These authors contributed equally to this work.

(C) Maayan Waldman, et al., 2016; Published by Mary Ann Liebert, Inc. This Open Access article is distributed under the terms of the Creative Commons Attribution Noncommercial License (http://creativecommons.org/licenses/by-nc/4.0/) which permits any noncommercial use, distribution, and reproduction in any medium, provided the original author(s) and the source are credited. 
expression decreases adipocyte heme and subsequently attenuates (hypertrophy) adipogenesis [10]. This reduction in adipogenesis is associated with an increase in the levels of EETs [13], which increase hematopoietic stem cell transplant engraftment that is associated with activation of the transcriptional factor activator protein 1 (AP-1) [14]. The AP-1 binding site is present in the human HO-1 promoter that activates HO-1 gene expression [15]. Therefore, the mechanism by which EET increases HO-1 expression may not be related solely to inhibition by the BTB and CNC homology 1, basic leucine zipper transcription factor 1 (Bach 1) [16]. EET affects adipocyte differentiation and hypertrophy both in vitro and in vivo $[7,17,18]$ by reprogramming the adipocyte phenotype.

The development of adipocytes in mice and humans follows a clearly defined pathway (adipogenesis) that begins with a common mesenchymal stem cell (MSC) that is pluripotent [19]. The early steps of the pathway leading to the generation and the commitment of MSCs to adipocyte lineage are unknown. Hypothetically, the determination of the fate of MSCs occurs early in the stages of cell differentiation ("commitment") and involves the interplay of intrinsic (genetic) and environmental (local and systemic) conditions to ultimately define cell fate. Factors such as those discussed earlier, that determine MSC proliferation and differentiation, also govern early adipocyte development and function. Currently, little is known about this process-from MSC to preadipocytes to adipocyte differentiation.

During adipogenesis, MSCs or preadipocytes differentiate into lipid-laden and insulin-sensitive adipocytes [20]. The acquisition of adipocyte phenotype and development of adipocyte function is characterized by chronological changes in the expression of multiple genes. Briefly, the stages of adipocyte differentiation are as follows: (1) MSC-derived adipocyte growth arrest, (2) clonal expansion, (3) a second stage of growth arrest or early differentiation, and (4) terminal differentiation-development of a mature adipocyte phenotype $[21,22]$. Concurrently as cells proceed to differentiation, there is an increase in transcription or de novo expression of several genes, including adiponectin, Glut 4, insulin receptor, and fatty acid synthase [21]. In the early phase of differentiation, preadipocyte cells are morphologically similar to fibroblasts. After clonal expansion, continued induction of adipogenesis leads to a drastic change in cell shape. Preadipocytes convert to a spherical shape; lipid droplets accumulate, and the preadipocyte progressively acquires the morphological and biochemical characteristics of a mature adipocyte, followed by triglyceride accumulation [22].

Administration of EET or inhibitors of $\mathrm{sEH}$ to obese mice is associated with a decrease in visceral subcutaneous fat and an increase in insulin sensitivity [23-26]. The CYP2J2mediated increase in EET increases fatty acid oxidation and adiposity $[17,27,28]$. Although EETs exhibit potent biological effects, including vasodilatation and inhibition of the inflammatory response [4,29,30] and in adiposity [17,27,28], their influence on mitochondrial function and proliferatoractivated receptor gamma coactivator- $1 \alpha(\mathrm{PGC}-1 \alpha)$ in relation to adipogenesis remains unknown.

Peroxisome PGC- $1 \alpha$ was originally described as a coactivator of peroxisome proliferator-activated receptor-gamma (PPAR- $\gamma$ ) that modulates expression of uncoupling protein 1 (UCP-1) and thermogenesis in brown fat [31,32]. PGC-1 $\alpha$ controls mitochondrial biogenesis and oxidative metabolism in many tissues, including brown adipose tissue $[11,33]$. Transgenic mice with mildly elevated muscle PGC- $1 \alpha$ are resistant to age-related obesity and diabetes resulting in a prolongation of their life span, suggesting that PGC- $1 \alpha$ influences the secretion of factors of biological influence that affect the function of other tissues [34]. Adipose-specific PGC- $1 \alpha$ deficiency results in a decrease in mitochondrial biogenesis and an increase in fatty acid oxidation, glucose, and insulin resistance [35].

In the present study, we examined whether the EETmediated regulation of adiposity is due to activation of PGC- $1 \alpha$ and an increase in UCP- 1 , HO- 1 , and $\beta$-catenin, resulting in decreased adipocyte terminal differentiation through the regulation of heme levels, essential for adipocyte proliferation and differentiation.

\section{Materials and Methods}

\section{Cell culture}

3T3-L1 mouse preadipocytes were purchased from the ATCC (ATCC, Manassas, VA). After thawing, 3T3-L1 cells were resuspended in an $\alpha$-minimal essential medium ( $\alpha$-MEM; Invitrogen, Carlsbad, CA) supplemented with $10 \%$ heat-inactivated fetal bovine serum (FBS; Invitrogen) and $1 \%$ antibiotic/antimycotic solution (Invitrogen). The cultures were maintained at $37^{\circ} \mathrm{C}$ in a $5 \% \mathrm{CO}_{2}$ incubator, and the medium was changed after $48 \mathrm{~h}$ and every 3-4 days thereafter. When the 3T3-L1 cells were confluent, the cells were recovered by the addition of $0.25 \%$ trypsin/EDTA (Invitrogen) and subcultured. For experiments, 3T3-L1 cells were seeded in 96- and 24-well plates at a density of 10,000 cells $/ \mathrm{cm}^{2}$ and cultured in $\alpha$-MEM with $10 \%$ FBS until $90 \%$ confluence was achieved. The medium was then replaced with adipogenic medium, and the cells were cultured for an additional 7 days. The adipogenic medium consisted of Dulbecco's modified Eagle's medium (DMEM) with high glucose (Invitrogen) supplemented with $10 \%$ (v/v) FBS, $10 \mu \mathrm{g} / \mathrm{mL}$ insulin (Sigma-Aldrich, St. Louis, MO), $0.5 \mathrm{mM}$ dexamethasone (Sigma-Aldrich), and $0.1 \mathrm{mM}$ indomethacin (Sigma-Aldrich). For some experiments, cells were treated with CoPP (Cobalt protoporphyrin; Frontier Scientific, Logan, UT) at a final concentration of $2-5 \mu \mathrm{M}$, as indicated in the figure legends; for 5-7 days, the EET analog, EET-A [(S)-2-(11-(nonyloxy) undec-8(Z)-enamido) succinic acid], was treated at a final dose of $1-10 \mu \mathrm{M}$, as indicated in the figure legends. To examine the effect of inhibition of $\mathrm{HO}$ activity on EET-A-treated cell, SnMP $(2 \mu \mathrm{M}$, Tin mesoporphyrin; Frontier Scientific) was added for the duration of EET-A treatment. Cells and conditioned media were collected after 7 days, as indicated in the figure legends.

At the experimental endpoints, cells were collected by trypsinization, washed once with PBS, and then lysed for both protein measurement and RNA extraction.

\section{Isolation and development of PGC-1 $\alpha$-deficient adipocytes}

SMART vector lentiviral shRNA-PPARGC1A or scrambled RNA (Dharmacon, Lafayette, CO) was applied to 3T3-L1 cells to establish a stably transduced cell line. Briefly, $1 \times 10^{6}$ cells were seeded in six-well plates 1 day before transduction. 
On the day of transduction, the transduction medium was made by $1 \times 10^{6}$ transducing units of lentiviral particles with $0.5 \mathrm{~mL} \alpha$-MEM growth medium applied to each well and incubated for $3 \mathrm{~h}$ to maximize the contact between each cell and lentiviral particles. Cells were also treated with the transduction medium without lentiviral particles, which served as untransduced control. Growth medium $(1.5 \mathrm{~mL})$ was then added to each well in the presence of $8 \mu \mathrm{g} / \mathrm{mL}$ polybrene (final concentration). After 48-h incubation, antibiotic selection medium ( $\alpha$-MEM growth medium with $10 \mu \mathrm{g} / \mathrm{mL}$ puromycin) was used to kill all the untransduced cells for either lentiscramble or cells treated with lentiviral shRNA-PPARGC1A. The growth medium was replaced every 2 days and used for differentiation and experimental studies.

\section{Oil Red O staining}

$0.21 \%$ Oil Red O in 100\% isopropanol (Sigma-Aldrich) was used for staining, as previously described [18]. Briefly, adipocytes were fixed in $10 \%$ formaldehyde, stained with Oil Red O for $10 \mathrm{~min}$, and rinsed with 60\% isopropanol (SigmaAldrich), where after the Oil Red $\mathrm{O}$ was eluted by incubation in $100 \mu 1100 \%$ isopropanol for $10 \mathrm{~min}$ and then the OD read at $490 \mathrm{~nm}$ for $0.5 \mathrm{~s}$, as previously described [7,18].

Lipid droplet size analysis. The lipid droplet size and number were acquired by analyzing the whole bottom surface area of an individual well of a 96-well plate. Each experiment was performed in triplicate, and the droplet analysis was performed [G]. The photographs are representative images of the experimental conditions. Cell size was measured using an ImagePro Analyzer (MediaCybernetics, Inc., Rockville, MD). The classification of the size of lipid droplets was based on the size of the two-dimensional area and expressed as pixels as we previously described [6].

\section{Flow cytometer measurement of MitoSox}

Mitochondrial superoxide formation was determined by the uptake of MitoSox ${ }^{\mathrm{TM}}$ Red reagent (Invitrogen) in live cells, as previously described [36]. Briefly, cells were plated at a density of 10,000 cells $/ \mathrm{cm}^{2}$ in six-well plates, with or without EET-A, $10 \mu \mathrm{M}$, overnight and then incubated with Hoechst nuclear stain $(5 \mu \mathrm{g} / \mathrm{mL})$ for $30 \mathrm{~min}$ in complete medium. The medium was removed, and the cells were incubated with MitoSox Red reagent $(5 \mu \mathrm{M})$ in Hank's balanced salt solution for $10 \mathrm{~min}$ at $37^{\circ} \mathrm{C}$ protected from light. The cells were then washed twice with PBS, collected by trypsinization, and finally washed with PBS supplemented with $2 \%$ bovine serum albumin (BSA; Fisher Scientific, Pittsburg, PA). The cells were then fixed in $2 \%$ formaldehyde solution for $10 \mathrm{~min}$, collected by centrifugation, and resuspended in PBS supplemented with 2\% BSA. Intensity of cellular fluorescence was measured using a Beckman Coulter's MoFlo XDP flow cytometer (Beckman Coulter, Indianapolis, IA) and analyzed with Kaluza v1.3 software (Beckman Coulter). More than 16,000 cells were measured per sample. Data are presented as a frequency distribution graph of fluorescence intensity of individual cells plotted on a logarithmic coordinate; the mean and SD are shown per individual sample. The mean values from triplicate cultures were used to calculate statistical difference between the control and EET-A-treated cultures.

\section{HPLC measurement of mitochondria-derived ROS and heme measurement}

Mitochondrial superoxide formation was determined by the uptake of MitoSox Red reagent (Invitrogen) in live cells, as previously described [37,38]. Briefly, cells were plated at a density of 10,000 cells $/ \mathrm{cm}^{2}$ in six-well plates with or without EET-A, $10 \mu \mathrm{M}$, overnight and then incubated with the MitoSox Red reagent $(5 \mu \mathrm{M})$ in Hank's balanced salt solution for $10 \mathrm{~min}$ at $37^{\circ} \mathrm{C}$ protected from light. The cells were washed twice with PBS, and $300 \mu \mathrm{L}$ acetonitrile was then added per well, followed by incubation on ice for $5 \mathrm{~min}$ protected from light. Cells were collected by scraping, and the lysate was transferred into microcentrifuge tubes. Samples were incubated at $-20^{\circ} \mathrm{C}$ for $1 \mathrm{~h}$, then pelleted by centrifugation at $14,000 \mathrm{~g}$ for $10 \mathrm{~min}$, and used for protein measurement, and the supernatant was then collected and stored at $-25^{\circ} \mathrm{C}$ until analyzed for MitoSox fluorescence (excitation/emission 510/580) using an HPLC system with a Jasco FP-1520 fluorescence detector and a Beckman ultrasphere reverse column $(\mathrm{C} 18)(5 \mu, 250 \times 4.6 \mathrm{~mm})$, and the values obtained were normalized to protein levels. Free heme and degraded heme protein, including myoglobin, were measured using a BioVision ELISA Kit (BioVision, Inc., Milpitas, CA).

\section{Cytokine measurements}

Adiponectin (high molecular weight, HMW), interleukin 6 (IL-6), and IL- $1 \alpha$ were determined in conditioned medium using an ELISA Assay ELISA Kit (R\&D Systems, Inc., Minneapolis, MN) according to the manufacturer's instructions and as previously described [7,39].

\section{Immunoblotting of signaling proteins}

Cells were trypsinized, pelleted by centrifugation, and lysed with lysis buffer supplemented with protease and phosphatase inhibitors (complete ${ }^{\mathrm{TM}}$ Mini and PhosSTOP ${ }^{\mathrm{TM}}$; Roche Diagnostics, Indianapolis, IA). Protein levels were quantified using a commercial assay (Bio-Rad, Hercules, CA). Protein samples were applied to sodium dodecyl sulfate (SDS) polyacrylamide gel (10\%-15\%), electrophoresed under denaturing conditions, and electrotransferred onto PVDF Immobilon-P membrane (Amersham Pharmacia, Piscataway, NJ) using a semidry transfer apparatus (BioRad). Membranes were blocked with Odyssey ${ }^{\circledR}$ Blocking Buffer (TBS) (LI-COR, Lincoln, NE) for $1 \mathrm{~h}$ at room temperature. Primary antibodies (1:500-1:1,000 dilution) in blocking buffer supplemented with $0.1 \%$ Tween 20 (Fisher Scientific) were incubated overnight at $4^{\circ} \mathrm{C}$, washed in Tris-buffered saline (TBS) supplemented with $0.1 \%$ Tween 20 , and then incubated with the appropriate fluorophore-conjugated secondary antibodies (1:5,0001;20,000) (LI-COR). Detection was completed with a LI-COR Odyssey instrument (LI-COR), and quantification of signals was completed with the Odyssey program (LI-COR).

\section{Quantitative real-time PCR}

Briefly, total RNA was extracted from pelleted cells using TRIzol $^{\circledR}$ (Ambion, Austin, TX) according to the manufacturer's 
instructions. Concentration of total RNA was determined by the use of a Take ${ }^{\circledR}$ plate and a Biotek ${ }^{\circledR}$ Plate Reader (Biotek, Winooski, VT). cDNA was synthesized from $1 \mu \mathrm{g}$ total RNA and 18s using the High-Capacity cDNA Reverse Transcription Kit (Applied Biosystems, Foster City, CA) according to the manufacturer's protocol, and PCR mRNA calculation was adjusted to $18 \mathrm{~s}$ as control. Quantitative real-time PCR analysis was performed using the 7500 Fast Real-Time PCR System (Applied Biosystems). The gene expression analysis levels were determined using the relative expression method with the threshold crossing point (Ct-value) as basis and as described [40].

\section{Statistical analysis}

The treatment groups were compared with the control group, and the experiments were analyzed using one-way analyses of variance (ANOVA) with Bonferroni tests for multiple comparisons. Data are presented as mean \pm SE. Statistical significance was defined as $P<0.05$.

\section{Results}

\section{EET reduces adipogenesis in differentiated adipocytes}

We examined the effect of CoPP on HO-1 induction and EET-A on adipocyte function by measuring the total amount of lipids. Differentiated adipocyte cells treated with either EET-A or CoPP display reduced large lipid droplet content compared with differentiated control cells $(P<0.05)$, an effect that was completely prevented by concomitant treatment with EET-A and an inhibitor of HO activity, SnMP (Fig. 1A). The results are quantitated in Fig. 1B. The effect of EET-A on adipogenesis was determined by counting cells with small lipid droplets in the cytoplasm and compared with large adipocytes (Fig. 1B). The percentage of cells with small lipid droplets increased in cells treated with EET. In differentiated cells treated with either EET or EET-SnMP and compared with untreated cells, the percentage of small and large lipid droplets is displayed in Fig. 1B, left and right panels, respectively. EET-A decreased adipocyte terminal
A
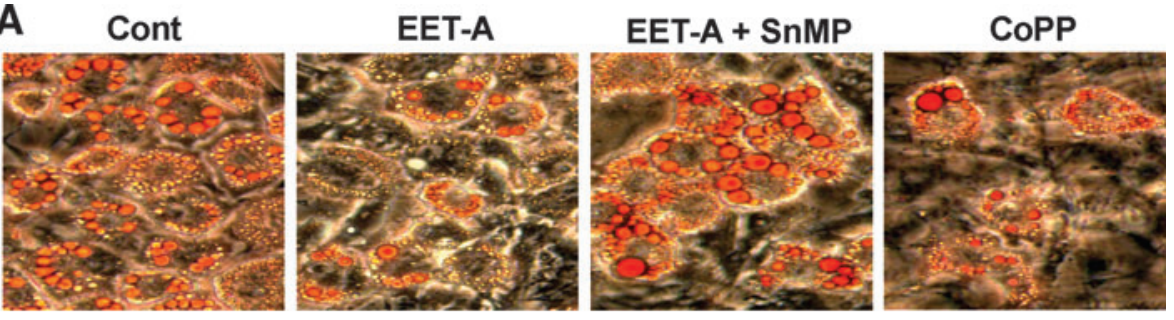

B


C


FIG. 1. EET-A and CoPP increase small adipocyte number and inhibit adipocyte terminal differentiation. 3T3-L1 cells cultured in differentiation medium were stained with Oil Red O. (A) Representative images of differentiated control cells and cells treated with EET-A $(1 \mu \mathrm{M})$, EET-A $(1 \mu \mathrm{M})+\operatorname{SnMP}(5 \mu \mathrm{M})$, and $\mathrm{CoPP}$ $(2 \mu \mathrm{M})$. Analysis of lipid droplet size (B). ${ }^{*} P<0.05$ versus control. (C) Representative blot and densitometry analysis of PGC- $1 \alpha$ protein levels in the presence of EET $(1 \mu \mathrm{M})$ with or without the addition of SnMP $(5 \mu \mathrm{M}) .{ }^{*} P<0.05$ versus control, $n=3$. (D) PGC- $1 \alpha$ mRNA levels in the presence of EET-A $(10 \mu \mathrm{M})$ with or without the addition of SnMP $(5 \mu \mathrm{M}) . * P<0.05$ versus control, $n=3$. EET-A, epoxyeicosatrienoic acid; PGC- $1 \alpha$, proliferation-activated receptor $\gamma$ coactivator $\alpha$. 
FIG. 2. Inflammatory and adipogenesis markers. The proinflammatory cytokines IL-1 $\alpha$ (A) and IL-6 (B) were measured by ELISA in the conditioned medium of treated and nontreated adipocytes at 7 days of differentiation and treated with EET-A and SnMP. (C) Adiponectin (HMW) levels in differentiated 3T3 cells treated with EET-A $(1 \mu \mathrm{M})$. Adiponectin levels secreted into the conditioned medium were measured using ELISA, $n=3-5$ in each group, $* P<0.05, \quad{ }^{\#} P<0.05$, and $* * P<$ 0.05 versus EET-A $(1 \mu \mathrm{M})$. EET-A, epoxyeicosatrienoic acid; IL-6, interleukin 6.

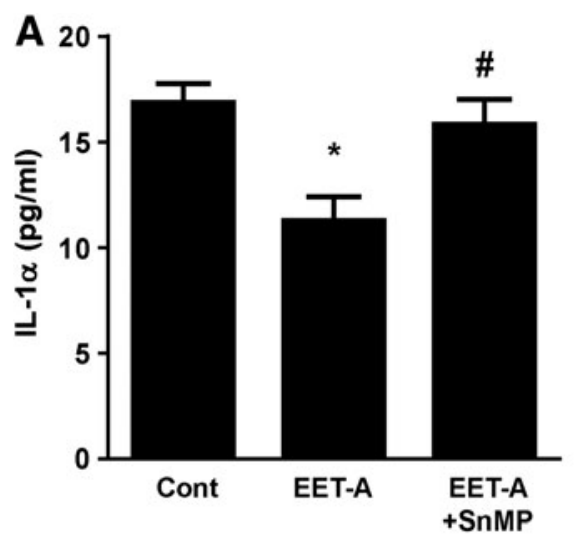

B

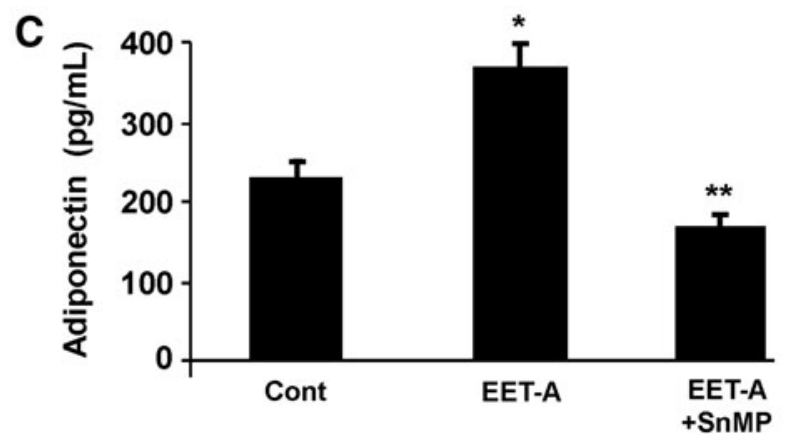

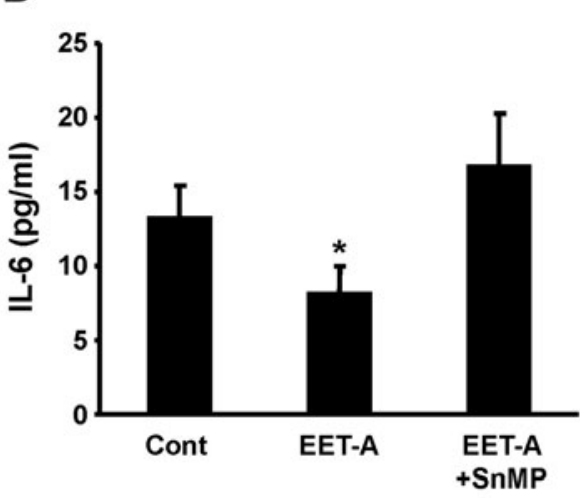

SnMP differentiation, that is, prevented the conversion of small adipocytes to large adipocytes (Fig. 1B). The adipocyte cell size in the absence of the EET-A agonist was $180 \pm 3$ pixels compared with $100 \pm 2.1$ pixels in the presence of EET-A (Fig. 1B, right panel).

We examined the levels of PGC- $1 \alpha$ in adipocytes treated with EET-A or SnMP. As seen in Fig. 1C, EET-A increased PGC-1 $\alpha$ levels, while SnMP largely prevented this effect. These results were confirmed by the levels of PGC- $1 \alpha$ mRNA levels (Fig. 1D).

\section{EET decreases inflammation during differentiation of adipocytes}

Treatment with EET-A reduced the levels of both IL- $1 \alpha$ and IL-6 compared with control, and SnMP blocked this effect (Fig. 2A, B). Adiponectin levels in the conditioned medium of adipocytes treated with EET-A were higher $(P<0.05)$ compared with untreated adipocytes. SnMP blocked the effect of EET-A $(P<0.05)$ (Fig. 2C).

\section{HO-1 expression is impaired in adipocytes deficient in PGC-1 $\alpha$}

The expression of PGC- $1 \alpha$ protein in the PGC- $1 \alpha \mathrm{KD}$ cells was $30 \%-35 \%$ of PGC- $1 \alpha$ protein levels in WT cells (Fig. 3A, B). Since EET increased PGC-1 $\alpha$ levels (Fig. 2) and EET is known to increase HO-1 protein expression, we measured HO-1 in WT, Mock, and PGC- $1 \alpha$ KD adipocyte cells treated with EET-A. In WT and Mock adipocyte cells, EET increased HO-1 levels (Fig. 3C). However, in PGC-1 $\alpha$ $\mathrm{KD}$ adipocyte cells, EET failed to increase the expression of HO-1, suggesting that PGC- $1 \alpha$ is necessary for HO-1 expression (Fig. 3D).

\section{PGC-1 $\alpha$ knockdown decreases mitochondrial viability}

Mitochondrial superoxide levels were elevated in PGC$1 \alpha$-deficient cells compared with WT cells (Fig. 4A, B), and EET-A significantly reduced mitochondrial superoxide levels in WT cells $(P<0.05)$ (Fig. 4C). UCP-1 protein levels were lower in PGC-1 $\alpha$-deficient cells compared with those in WT cells (Fig. 4D). Treatment with EET-A increased UCP-1 protein levels in WT cells $(P<0.05)$ but did not affect the UCP-1 levels in PGC- $1 \alpha$-deficient cells (Fig. 4D). Adipocyte cell heme levels were higher in the WT control compared with the EET-A-treated cells (Fig. 4E).

\section{PGC-1 $\alpha$ knockdown prevents EET-mediated $\mathrm{HO}-1$ induction}

To examine if EET induction of HO-1 is mediated through PGC- $1 \alpha$, preadipocytes were transfected with PGC$1 \alpha$ shRNA. As seen in Fig. 5A and B, the EET-mediated increase in HO- 1 protein was abolished in PGC- $1 \alpha$-deficient cells, suggesting that EET-A increases HO-1 through the direct effect of PGC-1 $\alpha$. As shown in Fig. 5C and D, the levels of manganese superoxide dismutase (MnSOD) are threefold lower in PGC-1 $\alpha$-deficient adipocyte cells compared with WT cells. Moreover, cells treated with EET-A displayed an increased level of MnSOD protein in both WT cells and PGC-1 $\alpha$-deficient cells (Fig. 5C, D).

\section{Effect of EET-A on $\beta$-catenin and Wnt10b}

We examined whether expression of $\beta$-catenin and wingless-type MMTV integration site family, member $10 \mathrm{~b}$ (Wnt10b) is dependent on the levels of PGC-1 expression by 
A

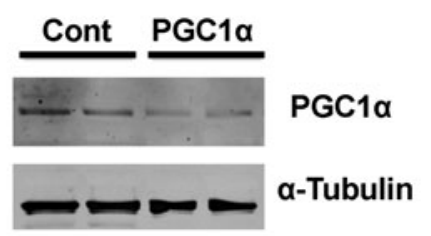



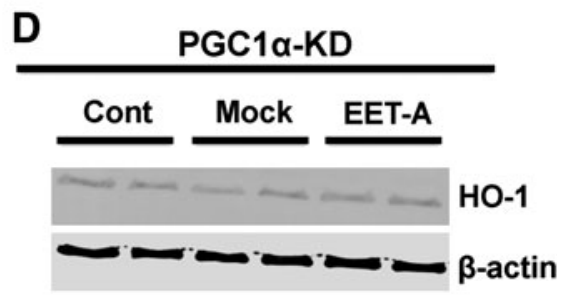

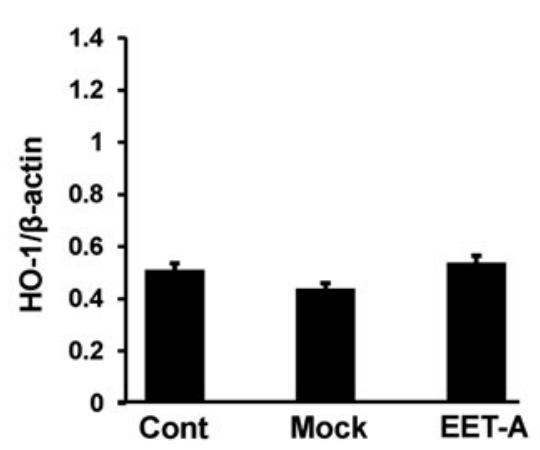
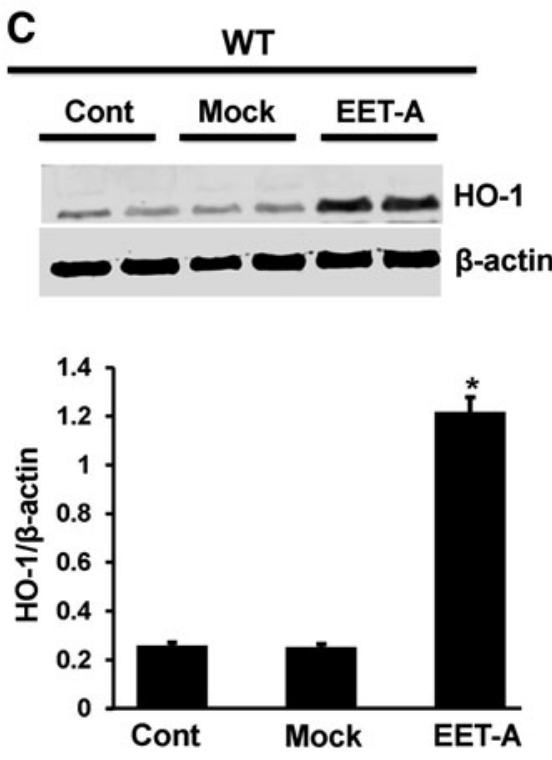

FIG. 3. (A and B) PGC- $1 \alpha$ protein is reduced in the 3T3-L1 preadipocyte. Data are shown as mean band density normalized to $\beta$-actin (mean $\pm \mathrm{SE}$; $n=3 ; * P<0.05$ compared to WT). (C and $\mathbf{D})$ The effect of EET on HO-1 expression in WT and PGC- $1 \alpha$ KD adipocyte cells was compared. Fully differentiated cells were treated with $1 \mu \mathrm{M}$ EET-A for 2 days in WT, Mock, and PGC- $1 \alpha$ KD cells. Cells were lysed, and protein expression was determined $(* P<0.01$ compared to WT, $n=3)$. EET-A, epoxyeicosatrienoic acid; PGC- $1 \alpha$, proliferation-activated receptor $\gamma$ coactivator $\alpha$.
EET. Treatment of adipocyte cells with EET-A significantly $(* P<0.05)$ increased both $\beta$-catenin and Wnt10b mRNA expression levels compared with control (Fig. 6A, B), suggesting the involvement of HO-1-PGC-1 signaling in regulation of stage of differentiations of mouse adipocytes.

\section{Effect of EET-A on MEST mRNA and protein levels}

To further characterize the effect of EET-A on adipocytes during maturation, we examined the levels of mesodermspecific transcript/paternally expressed gene 1 protein (MEST/Peg1). As seen in Fig. 7A and B, treatment of adipocyte cells with EET-A $(10 \mu \mathrm{M})$ during differentiation for 7-8 days reduced MEST mRNA and protein levels compared with vehicle control cells.

\section{Discussion}

This is the first study to demonstrate that EET, a lipid mediator, is situated upstream of the PGC- $1 \alpha$ signaling cascade and plays a critical role in the regulation of adipocyte differentiation. Furthermore, this process involves an increase in HO-1 expression and inhibition of the progression toward terminal adipocyte differentiation. These novel findings highlighting EET-PGC- $1 \alpha$ interplay suggest that this plays a significant role in adipocyte proliferation, clonal expansion, growth arrest, and differentiation. The transcriptional coactivator PGC- $1 \alpha$, first identified as a regulator of PPAR- $\gamma$ in brown adipose tissue rich in the mitochondria, has a special role in thermogenesis [31]. PGC- $1 \alpha$ is highly expressed in tissues with a high energy demand, such as the heart, skeletal muscle, and brown adipose tissue, where it plays a critical role in maintaining mitochondrial biogenesis and function and as well as cellular energy metabolism $[11,41]$. In PGC-1 $\alpha$-deficient cells, HO-1 levels are reduced compared with wild-type cells or Mock cells (Fig. 3C, D). This novel finding adds support to our report that PGC- $1 \alpha$ is essential for the EET-mediated increase in HO-1 expression and a critical role for PGC- $1 \alpha$ in reducing adipogenesis by controlling heme levels. These results identify PGC- $1 \alpha$ as a key component of the regulatory pathway involved in the control of mitochondria-derived ROS and adiponectin release. PGC- $1 \alpha$ expression is increased in response to physiological stimuli that demand increased mitochondrial energy production, such as exercise and fasting [42-45], and may play an important role in the development of antiobesity therapies.

Exogenous EET reduced clonal expansion of adipocytes, thereby favoring healthy small adipocytes, as opposed to hypertrophic lipid accumulation. Protection against adipose tissue dysfunction, including preservation of anti-inflammatory adipokines such as adiponectin, is related to an adipocyte of smaller size $[7,10,23,46]$. The beneficial effect of EET-A was blocked by SnMP, indicating that EET is working through the HO system, which controls the cellular levels of heme, leading 
FIG. 4. (A) Representative plot showing flow cytometry analysis of mitochondrial superoxide levels in WT (left panel) and PGC-1 $\alpha$-deficient (right panel) undifferentiated adipocytes and (B) statistical analysis of the integrated MitoSox ${ }^{\mathrm{TM}}$ fluorescence intensity, $n=3$, $* P<0.05$ compared to WT. (C) Mitochondrial superoxide levels in WT adipocytes as measured by MitoSox incorporation and analysis of HPLC, $n=3, \quad * P<0.05$ versus control. (D) Representative western blot showing UCP-1 protein levels in WT and PGC$1 \alpha$-deficient cells (top) and the densitometry analysis (bottom), $n=3, * P<$ 0.05 compared to control. (E) Measurement of adipocyte heme levels, $n=3, * P<0.05$ compared to control. PGC-1 $\alpha$, proliferation-activated receptor $\gamma$ coactivator $\alpha$; UCP-1, uncoupling protein 1 .
A

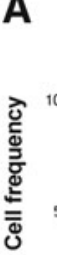


WT

PGC-1 $\alpha$ KD



B $\square$ wT

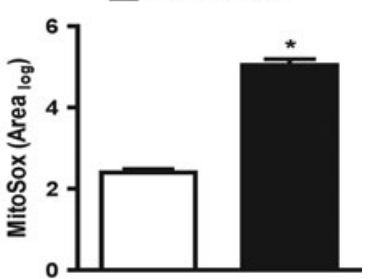

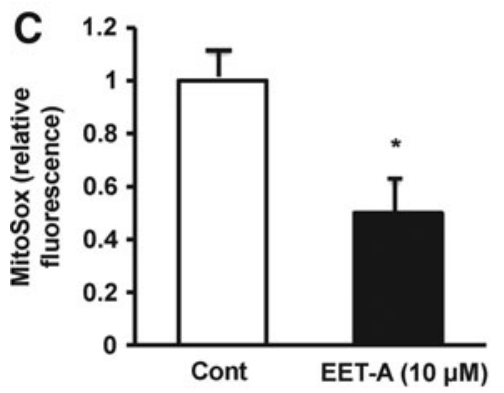

D
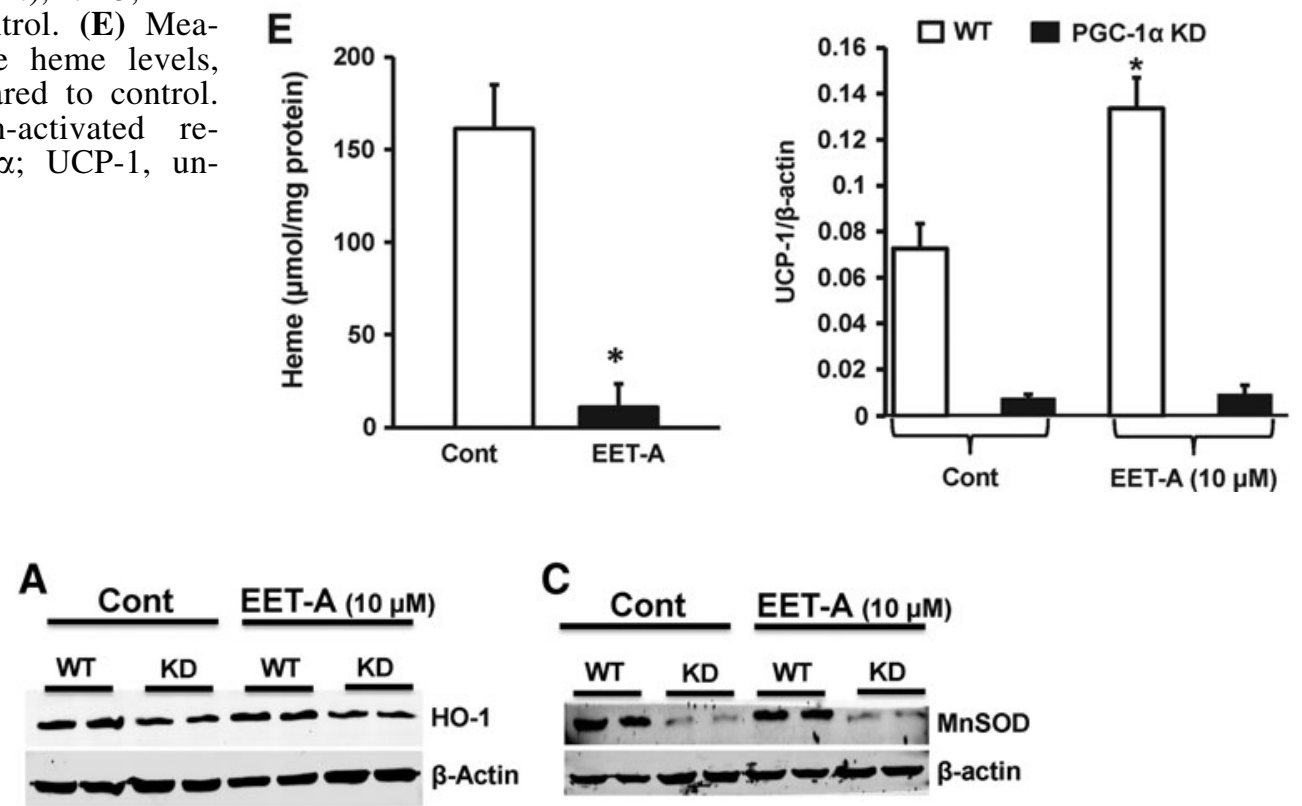

B


FIG. 5. Western blot analysis for the expression of the HO- 1 protein in WT and PGC-1 $\alpha$-deficient cells with and without EET-A $(10 \mu \mathrm{M})$ treatment. Representative blots of HO-1 and $\beta$-actin (A) and densitometry analysis of HO- 1 protein levels, normalized to $\beta$-actin in untreated (control) and cells treated for 7 days with EET-A, added every other day (B). Western blot analysis of the expression of the antioxidant protein MnSOD in differentiated adipocytes. WT- and PGC-1 $\alpha$-silenced cells with and without EET-A $(10 \mu \mathrm{M})$ treatment. Representative blots of MnSOD and $\beta$-actin (C) and densitometry analysis of MnSOD protein levels, normalized to $\beta$-actin in untreated (control) and cells treated for 7 days or $16 \mathrm{~h}$ after EETA supplementation for protein measurement and mRNA, respectively, following treatment with EET-A (D). $n=3, * P<0.05$ compared to WT; $* * P<0.01$ compared to WT EET-A treated. EET-A, epoxyeicosatrienoic acid; HO-1, heme oxygenase-1; MnSOD, manganese superoxide dismutase; PGC-1 $\alpha$, proliferation-activated receptor $\gamma$ coactivator $\alpha$. 

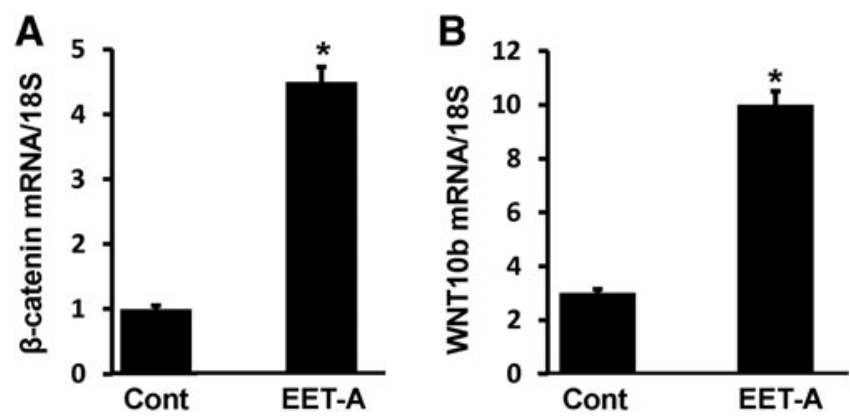

FIG. 6. Effect of EET-A $(10 \mu \mathrm{M})$ on expression of $\beta$-catenin and Wnt10b, mRNA, and/or protein levels. (A) $\beta$-catenin mRNA levels. (B) Wnt10b mRNA levels, $n=3, * P<0.05$ versus control. EET-A, epoxyeicosatrienoic acid; Wnt10b, wingless-type MMTV integration site family, member $10 \mathrm{~b}$.

to terminal adipocyte stem cell differentiation and enlargement. It is clear that PGC- $1 \alpha$ regulates heme levels by two mechanisms: by increased $5^{\prime}$-aminolevulinate synthase (ALAS, the rate-limiting enzyme of heme biosynthesis) and heme levels needed for full differentiation; simultaneously, PGC- $1 \alpha$ increases HO- 1 as a result of EET treatment that reduces heme levels and prevents terminal adipocyte differentiation. SnMP increases levels of cellular heme by inhibiting heme oxygenase, the rate-limiting enzyme in heme degradation [47]. We report here that EET increases PGC-1 $\alpha$, an effect not blocked by SnMP. This confirms previous studies showing that PGC- $1 \alpha$ is particularly sensitive to intracellular heme levels, particularly via Rev-Erb $\alpha$ regulation [12], and plays a significant role in the increase in the levels of heme by upregulation of ALAS [48]. However, EET-mediated increase in PGC- $1 \alpha$ leading to increased levels of HO- 1 also reduced heme to control adipocyte cells during terminal differentiation. Interestingly, SnMP increases cellular heme levels, while EET increases HO-1 via PGC-1 $\alpha$. Therefore, the net effect of SnMP treatment did not negatively impact PGC- $1 \alpha$ mRNA or protein levels when added to cells together with EET. This is in all probability due to the fact that EET decreases heme and SnMP increases heme, leaving PGC- $1 \alpha$ levels unchanged. We speculate that the EET-mediated increase in PGC- $1 \alpha$ affects adipocyte expansion and full differentiation by several

B

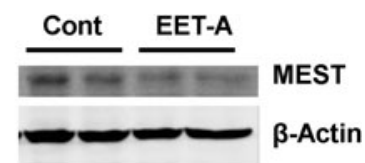

A


FIG. 7. Effect of EET-A $(10 \mu \mathrm{M})$ on MEST mRNA (A) and protein (B) levels after 7-8 days of differentiation. $n=3$, $* P<0.05$ versus control. MEST, mesoderm-specific transcript.

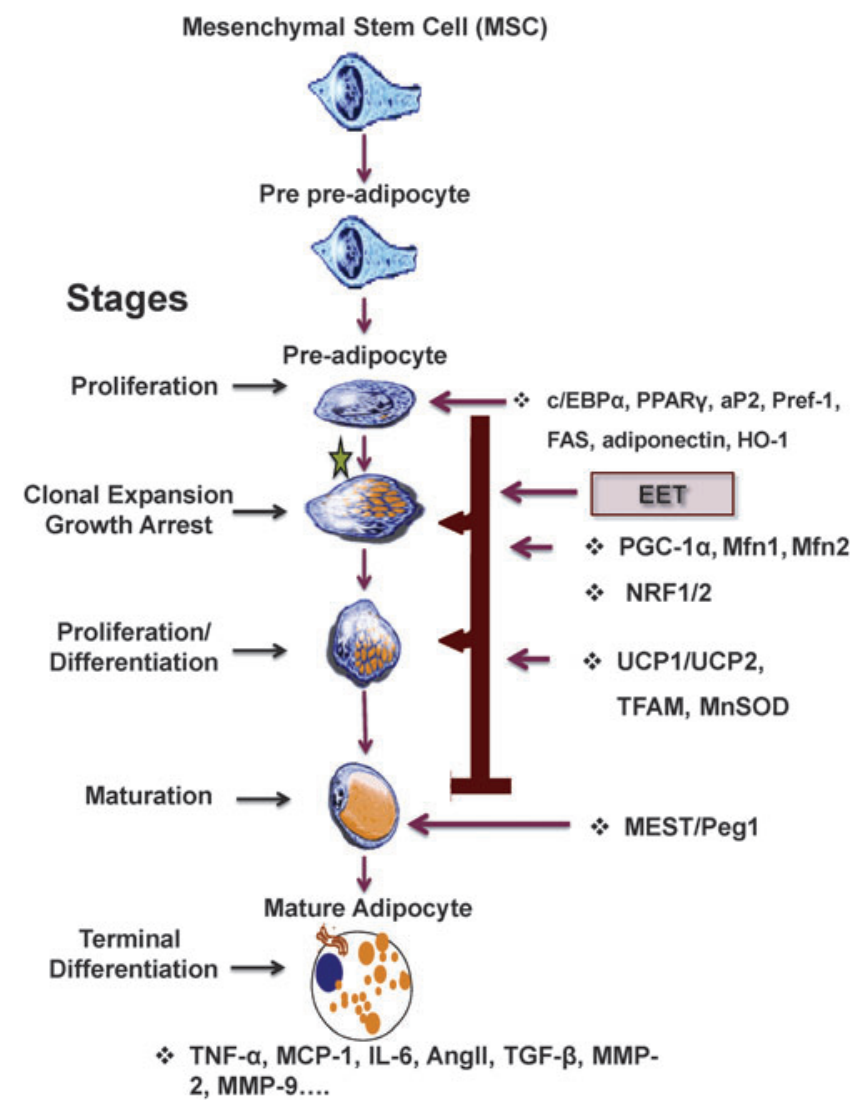

FIG. 8. Schematic presentation of the possible mechanism by which EET-PGC- $1 \alpha$ modulates pluripotent hematopoietic stem cell precursors that give rise to a mesenchymal precursor cell with the potential to differentiate along mesodermal lineages into myoblast, including adipocytes. When MSC is placed under appropriate adipogenic conditions, MSC begins to exhibit specific gene expression in preadipocytes that undergo proliferation, clonal expansion, and growth arrest. EET synthesis by Cyp2c44 increases PGC- $1 \alpha$ levels and translocation to the nucleus. In the nucleus together with PGC- $1 \alpha$ induced the expression of HO-1 and UCP-1. HO-1 reduces mitochondrial superoxide [55] and increases Cyp2c44 levels and activity. Arrows indicate that $\mathrm{PGC}-1 \alpha-\mathrm{HO}-1$ effect is present at 2-3 steps in the differentiation of MSC to terminally differentiated adipocytes. During this process, PGC- $1 \alpha$ increases HO- 1 expression, which is associated with a decrease in mitochondria-derived ROS (MitoSox). HO-1 gene activation resulted in reprogramming the adipocyte phenotype to express a new healthy phenotype, as evidenced by the expression of fatty acid oxidation enzymes, Wnt10b signaling, and release of adiponectin, while PGC- $1 \alpha$ increases the expression of thermogenic genes, including UCP-1, decreases MEST, and prevents cells entering into proinflammatory cytokines, increasing detrimental systemic effects on other organs. EET, epoxyeicosatrienoic acid; HO-1, heme oxygenase-1; MSC, mesenchymal stem cell; PGC$1 \alpha$, proliferation-activated receptor $\gamma$ coactivator $\alpha$; ROS, reactive oxygen species; UCP-1, uncoupling protein 1; Mfn1, Mfn2, mitochondrial fusion proteins-1 and 2.

mechanisms: (1) by acting upstream of PGC- $1 \alpha$ and by decreasing heme levels via induction of HO-1, (2) by increasing mitochondrial MnSOD and UCP-1, and (3) through a decrease in MEST/PEG1, necessary for terminal differentiation and adipocyte enlargement [7,13,18,23,28,49-52]. 
HO-1 has multiple cellular effects, including regulating bioavailability of heme and heme-containing denatured proteins [53], generation of bioactive metabolites-carbon monoxide (CO) and biliverdin (BV), preventing the cellular buildup of free (nonprotein bound) heme, and preventing free radical and mitochondrial dysfunction [53,54]. HO-1 plays a role in mitochondrial biogenesis and function and cytochrome oxidase $[55,56]$. The effect of PGC- $1 \alpha$ on mitochondrial function and adipogenesis is exemplified by the EET-A-mediated increase in Cyp2c44, leading to a new transcriptional role during adipogenesis, for example, by activation of PGC- $1 \alpha$ expression and reduction in mitochondrial ROS levels (Fig. 4). These results provide a new pathway comprising EET, PGC- $1 \alpha$, and HO- 1 that forms a positive feedback loop that stimulates mitochondrial function. Thus, EET, PGC- $1 \alpha$, and HO-1 appear to form a module that serves as a molecular "switch" to genetically reprogram the adipocyte phenotype to express lower levels of MEST (Fig. 7A, B) and prevent hypoadiponectinemia $[7,18]$ through upregulation of PGC- $1 \alpha$ and an increase in mitochondrial function and a decrease in MitoSox.

EET is the first lipid mediator derived from lipid metabolism via the CYP system to regulate PGC- $1 \alpha$. A dearth of EET is considered to be the leading promoter of adipocyte hypertrophy and dysfunction (large adipocyte). Cells treated with EET are associated with upregulation of PGC- $1 \alpha-\mathrm{HO}-1$, suggesting that EETs regulate adipocyte progression toward differentiation and adipogenesis through the activation of PGC- $1 \alpha$ signaling. Moreover, the protein level of the adipogenic MEST increased with differentiation (data not shown). Importantly, the MEST mRNA and protein levels were reduced by treatment of cells with EET-A during differentiation (Fig. 7).

We show here a direct link between EET-mediated activation of PGC- $1 \alpha$ and reduction in mitochondrial ROS production results in an increase in the number of small adipocytes. This novel finding demonstrates that EET is associated with epigenetic changes, that is, increases in PGC- $1 \alpha$, HO- 1 , and mitochondrial function not seen in terminally differentiated adipocytes. The EET-mediated increases in PGC- $1 \alpha$, including an increase in adiponectin expression and secretion and reduced secretion of proinflammatory cytokines resulting in a decrease in the number of the inflamed adipocyte phenotypes, appear to be dependent on intracellular heme levels as heme is essential for full differentiation of adipocyte [9,57,58]. These results are summarized in Fig. 8. During adipogenesis, MSCs or preadipocytes differentiate into lipid-laden and insulinsensitive adipocytes [20]. The acquisition of adipocyte phenotype and development of adipocyte function is characterized by chronological changes in the expression of multiple genes. Effect of drugs on programmed adipocyte cell differentiation may influence adipocytes to express adipogenic markers for maturation and terminal differentiation or downregulate such genes in favor of an increase in adiponectin and mitochondrial function. Adipose tissue and adipocyte cells play an important role in insulin resistance through the production and secretion of a variety of proteins, including tumor necrosis factor (TNF)- $\alpha$, IL-6, leptin, and adiponectin [59-62]. In contrast, terminal differentiation is associated with adipocyte enlargement and the expression of several markers, including Peg1/ MEST [7,18,50], and proinflammatory cytokines and suppression of adiponectin synthesis [18,63]. This process is highly regulated by the appearance of early, intermediate, and late mRNA/protein markers and triglyceride accumulation (Fig. 8). This suggests that EET rescues mitochondrial function by activation of PGC- $1 \alpha$ and that interplay between EET and PGC- $1 \alpha$ is inexorably linked in the regulation of HO-1, heme, and stage of adipocyte growth by modulating the level of gene expression that leads to adipocyte maturation and terminal differentiation.

\section{Acknowledgments}

This work was supported by the National Institutes of Health grants HL55601 and HL34300, the Brickstreet Foundation and the Huntington Foundation (NGA) and gifts from the Ablon, Lang and Renfield Foundations to The Rockefeller University (AK). We thank Jennifer Brown for her outstanding editorial assistance in the preparation of the article.

\section{Author Disclosure Statement}

All authors had full access to the data and take responsibility for its integrity. All authors have read and agreed with the article as written. There are no conflicts of interest among the authors.

\section{References}

1. Zeldin DC. (2001). Epoxygenase pathways of arachidonic acid metabolism. J Biol Chem 276:36059-36062.

2. Capdevila JH, JR Falck, and RC Harris. (2000). Cytochrome $\mathrm{P} 450$ and arachidonic acid bioactivation. Molecular and functional properties of the arachidonate monooxygenase. J Lipid Res 41:163-181.

3. Node K, Y Huo, X Ruan, B Yang, M Spiecker, K Ley, DC Zeldin, and JK Liao. (1999). Anti-inflammatory properties of cytochrome P450 epoxygenase-derived eicosanoids. Science 285:1276-1279.

4. Spiecker M and JK Liao. (2005). Vascular protective effects of cytochrome p450 epoxygenase-derived eicosanoids. Arch Biochem Biophys 433:413-420.

5. Vanella L, DH Kim, D Asprinio, SJ Peterson, I Barbagallo, A Vanella, D Goldstein, S Ikehara, A Kappas, and NG Abraham. (2010). HO-1 expression increases mesenchymal stem cell-derived osteoblasts but decreases adipocyte lineage. Bone 46:236-243.

6. Burgess AP, L Vanella, L Bellner, K Gotlinger, JR Falck, NG Abraham, ML Schwartzman, and A Kappas. (2012). Heme oxygenase (HO-1) rescue of adipocyte dysfunction in HO-2 deficient mice via recruitment of epoxyeicosatrienoic acids (EETs) and adiponectin. Cell Physiol Biochem 29:99-110.

7. Kim DH, L Vanella, K Inoue, A Burgess, K Gotlinger, VL Manthati, SR Koduru, DC Zeldin, JR Falck, ML Schwartzman, and NG Abraham. (2010). Epoxyeicosatrienoic acid agonist regulates human mesenchymal stem cell-derived adipocytes through activation of HO-1-pAKT signaling and a decrease in PPARgamma. Stem Cells Dev 19:18631873.

8. Chen JJ and IM London. (1981). Hemin enhances the differentiation of mouse 3 T3 cells to adipocytes. Cell 26:117122.

9. Kumar N, LA Solt, Y Wang, PM Rogers, G Bhattacharyya, TM Kamenecka, KR Stayrook, C Crumbley, ZE Floyd, et al. (2010). Regulation of adipogenesis by natural and synthetic REV-ERB ligands. Endocrinology 151:3015-3025. 
10. Abraham NG, JM Junge, and GS Drummond. (2016). Translational significance of heme oxygenase in obesity and metabolic syndrome. Trends Pharmacol Sci 37:17-36.

11. Wu Z, P Puigserver, U Andersson, C Zhang, G Adelmant, V Mootha, A Troy, S Cinti, B Lowell, RC Scarpulla, and BM Spiegelman. (1999). Mechanisms controlling mitochondrial biogenesis and respiration through the thermogenic coactivator PGC-1. Cell 98:115-124.

12. Wu N, L Yin, EA Hanniman, S Joshi, and MA Lazar. (2009). Negative feedback maintenance of heme homeostasis by its receptor, Rev-erbalpha. Genes Dev 23:2201-2209.

13. Burgess A, L Vanella, L Bellner, ML Schwartzman, and NG Abraham. (2012). Epoxyeicosatrienoic acids and heme oxygenase- 1 interaction attenuates diabetes and metabolic syndrome complications. Prostaglandins Other Lipid Mediat 97:1-16.

14. Li P, JL Lahvic, V Binder, EK Pugach, EB Riley, OJ Tamplin, D Panigrahy, TV Bowman, FG Barrett, et al. (2015). Epoxyeicosatrienoic acids enhance embryonic haematopoiesis and adult marrow engraftment. Nature 523:468-471.

15. Lavrovsky Y, ML Schwartzman, RD Levere, A Kappas, and NG Abraham. (1994). Identification of binding sites for transcription factors NF-kappa B and AP-2 in the promoter region of the human heme oxygenase 1 gene. Proc Natl Acad Sci U S A 91:5987-5991.

16. Sodhi K, N Puri, K Inoue, JR Falck, ML Schwartzman, and NG Abraham. (2012). EET agonist prevents adiposity and vascular dysfunction in rats fed a high fat diet via a decrease in Bach 1 and an increase in HO-1 levels. Prostaglandins Other Lipid Mediat 98:133-142.

17. Zha W, ML Edin, KC Vendrov, RN Schuck, FB Lih, JL Jat, JA Bradbury, LM Degraff, K Hua, et al. (2014). Functional characterization of cytochrome P450-derived epoxyeicosatrienoic acids in adipogenesis and obesity. J Lipid Res 55: 2124-2136.

18. Vanella L, K Sodhi, DH Kim, N Puri, M Maheshwari, TD Hinds, Jr., L Bellner, D Goldstein, SJ Peterson, JI Shapiro, and NG Abraham. (2013). Increased heme-oxygenase 1 expression decreases adipocyte differentiation and lipid accumulation in mesenchymal stem cells via upregulation of the canonical Wnt signaling cascade. Stem Cell Res Ther 4:28.

19. Vazquez-Vela ME, N Torres, and AR Tovar. (2008). White adipose tissue as endocrine organ and its role in obesity. Arch Med Res 39:715-728.

20. Lefterova MI and MA Lazar. (2009). New developments in adipogenesis. Trends Endocrinol Metab 20:107-114.

21. Rosen ED, CJ Walkey, P Puigserver, and BM Spiegelman. (2000). Transcriptional regulation of adipogenesis. Genes Dev 14:1293-1307.

22. Gregoire FM, CM Smas, and HS Sul. (1998). Understanding adipocyte differentiation. Physiol Rev 78:783-809.

23. Burgess A, M Li, L Vanella, DH Kim, R Rezzani, L Rodella, K Sodhi, M Canestraro, P Martasek, SJ et al. (2010). Adipocyte heme oxygenase-1 induction attenuates metabolic syndrome in both male and female obese mice. Hypertension 56:1124-1130.

24. Ndisang JF. (2010). Role of heme oxygenase in inflammation, insulin-signalling, diabetes and obesity. Mediators Inflamm 2010:359732.

25. Peterson SJ, G Drummond, DH Kim, M Li, AL Kruger, S Ikehara, and NG Abraham. (2008). L-4F treatment reduces adiposity, increases adiponectin levels, and improves insulin sensitivity in obese mice. J Lipid Res 49:1658-1669.
26. Nicolai A, M Li, DH Kim, SJ Peterson, L Vanella, V Positano, A Gastaldelli, R Rezzani, LF Rodella, et al. (2009). Heme oxygenase-1 induction remodels adipose tissue and improves insulin sensitivity in obesity-induced diabetic rats. Hypertension 53:508-515.

27. Zhang S, G Chen, N Li, M Dai, C Chen, P Wang, H Tang, SL Hoopes, DC Zeldin, DW Wang, and X Xu. (2015). CYP2J2 overexpression ameliorates hyperlipidemia via increased fatty acid oxidation mediated by the AMPK pathway. Obesity (Silver Spring) 23:1401-1413.

28. Abraham NG, K Sodhi, AM Silvis, L Vanella, G Favero, R Rezzani, C Lee, DC Zeldin, and Schwartzman ML. (2014). CYP2J2 targeting to endothelial cells attenuates adiposity and vascular dysfunction in mice fed a high-fat diet by reprogramming adipocyte phenotype. Hypertension 64:1352-1361.

29. Campbell WB. (2000). New role for epoxyeicosatrienoic acids as anti-inflammatory mediators. Trends Pharmacol Sci 21:125-127.

30. Omata K, K Abe, HL Sheu, K Yoshida, E Tsutsumi, K Yoshinaga, NG Abraham, and M Laniado-Schwartzman. (1992). Roles of renal cytochrome P450-dependent arachidonic acid metabolites in hypertension. Tohoku J Exp Med 166:93-106.

31. Puigserver $\mathrm{P}, \mathrm{Z}$ Wu, CW Park, R Graves, $\mathrm{M}$ Wright, and BM Spiegelman. (1998). A cold-inducible coactivator of nuclear receptors linked to adaptive thermogenesis. Cell 92: 829-839.

32. Bostrom P, J Wu, MP Jedrychowski, A Korde, L Ye, JC Lo, KA Rasbach, EA Bostrom, JH Choi, et al. (2012). A PGC1alpha-dependent myokine that drives brown-fat-like development of white fat and thermogenesis. Nature 481:463-468.

33. Puigserver P and BM Spiegelman. (2003). Peroxisome proliferator-activated receptor-gamma coactivator 1 alpha (PGC-1 alpha): transcriptional coactivator and metabolic regulator. Endocr Rev 24:78-90.

34. Wenz T, SG Rossi, RL Rotundo, BM Spiegelman, and CT Moraes. (2009). Increased muscle PGC-1alpha expression protects from sarcopenia and metabolic disease during aging. Proc Natl Acad Sci U S A 106:20405-20410.

35. Kleiner S, RJ Mepani, D Laznik, L Ye, MJ Jurczak, FR Jornayvaz, JL Estall, BD Chatterjee, GI Shulman, and BM Spiegelman. (2012). Development of insulin resistance in mice lacking PGC-1alpha in adipose tissues. Proc Natl Acad Sci U S A 109:9635-9640.

36. Li R, N Jen, F Yu, and TK Hsiai. (2011). Assessing mitochondrial redox status by flow cytometric methods: vascular response to fluid shear stress. Curr Protoc Cytom Chapter 9:Unit 9.37.

37. Infantino V, V Iacobazzi, A Menga, ML Avantaggiati, and F Palmieri. (2014). A key role of the mitochondrial citrate carrier (SLC25A1) in TNFalpha- and IFNgamma-triggered inflammation. Biochim Biophys Acta 1839:1217-1225.

38. Alhawaj R, D Patel, MR Kelly, D Sun, and MS Wolin. (2015). Heme biosynthesis modulation via delta-aminolevulinic acid administration attenuates chronic hypoxia-induced pulmonary hypertension. Am J Physiol Lung Cell Mol Physiol 308:L719-L728.

39. Sambuceti G, S Morbelli, L Vanella, C Kusmic, C Marini, M Massollo, C Augeri, M Corselli, C Ghersi, et al. (2009). Diabetes impairs the vascular recruitment of normal stem cells by oxidant damage; reversed by increases in pAMPK, heme oxygenase-1 and adiponectin. Stem Cells 27:399-407.

40. Pfaffl MW. (2001). A new mathematical model for relative quantification in real-time RT-PCR. Nucleic Acids Res 29:e45. 
41. St-Pierre J, J Lin, S Krauss, PT Tarr, R Yang, CB Newgard, and BM Spiegelman. (2003). Bioenergetic analysis of peroxisome proliferator-activated receptor gamma coactivators 1alpha and 1beta (PGC-1alpha and PGC-1beta) in muscle cells. J Biol Chem 278:26597-26603.

42. Baar K, AR Wende, TE Jones, M Marison, LA Nolte, M Chen, DP Kelly, and JO Holloszy. (2002). Adaptations of skeletal muscle to exercise: rapid increase in the transcriptional coactivator PGC-1. FASEB J 16:1879-1886.

43. Terada S and I Tabata. (2004). Effects of acute bouts of running and swimming exercise on PGC-1alpha protein expression in rat epitrochlearis and soleus muscle. Am J Physiol Endocrinol Metab 286:E208-E216.

44. H Pilegaard, B Saltin, and PD Neufer. (2003). Exercise induces transient transcriptional activation of the PGC-1alpha gene in human skeletal muscle. J Physiol 546:851-858.

45. Rhee J, Y Inoue, JC Yoon, P Puigserver, M Fan, FJ Gonzalez, and BM Spiegelman. (2003). Regulation of hepatic fasting response by PPARgamma coactivator-1alpha (PGC-1): requirement for hepatocyte nuclear factor 4alpha in gluconeogenesis. Proc Natl Acad Sci U S A 100:4012-4017.

46. Skurk T, C Alberti-Huber, C Herder, and H Hauner. (2007). Relationship between adipocyte size and adipokine expression and secretion. J Clin Endocrinol Metab 92:10231033.

47. Drummond GS and A Kappas. (1981). Prevention of neonatal hyperbilirubinemia by tin protoporphyrin IX, a potent competitive inhibitor of heme oxidation. Proc Natl Acad Sci U S A 78:6466-6470.

48. Handschin C, J Lin, J Rhee, AK Peyer, S Chin, PH Wu, UA Meyer, and BM Spiegelman. (2005). Nutritional regulation of hepatic heme biosynthesis and porphyria through PGC1alpha. Cell 122:505-515.

49. Peterson SJ, DH Kim, M Li, V Positano, L Vanella, LF Rodella, F Piccolomini, N Puri, A Gastaldelli, C Kusmic, L'Abbate A, and NG Abraham. (2009). The L-4F mimetic peptide prevents insulin resistance through increased levels of HO-1, pAMPK, and pAKT in obese mice. J Lipid Res 50:1293-1304.

50. Takahashi M, Y Kamei, and O Ezaki. (2005). Mest/Peg1 imprinted gene enlarges adipocytes and is a marker of adipocyte size. Am J Physiol Endocrinol Metab 288:E117-E124.

51. Vanella L, Li M, D Kim, G Malfa, L Bellner, T Kawakami, and Abraham NG. (2012). ApoA1: mimetic peptide reverses adipocyte dysfunction in vivo and in vitro via an increase in heme oxygenase (HO-1) and Wnt10b. Cell Cycle 11:706-714.

52. Vanella L, DH Kim, K Sodhi, I Barbagallo, AP Burgess, JR Falck, ML Schwartzman, and NG Abraham. (2011). Crosstalk between EET and HO-1 downregulates Bach1 and adipogenic marker expression in mesenchymal stem cell derived adipocytes. Prostaglandins Other Lipid Mediat 96:54-62.

53. Abraham NG and A Kappas. (2008). Pharmacological and clinical aspects of heme oxygenase. Pharmacol Rev 60: 79-127.

54. Balla J, HS Jacob, G Balla, K Nath, JW Eaton, and GM Vercellotti. (1993). Endothelial-cell heme uptake from heme proteins: induction of sensitization and desensitization to oxidant damage. Proc Natl Acad Sci U S A 90: 9285-9289.

55. Di Noia MA, DS Van, F Palmieri, LM Yang, S Quan, AI Goodman, and NG Abraham. (2006). Heme oxygenase-1 enhances renal mitochondrial transport carriers and cytochrome $\mathrm{C}$ oxidase activity in experimental diabetes. J Biol Chem 281:15687-15693.

56. Piantadosi CA, MS Carraway, A Babiker, and HB Suliman. (2008). Heme oxygenase-1 regulates cardiac mitochondrial biogenesis via Nrf2-mediated transcriptional control of nuclear respiratory factor-1. Circ Res 103:1232-1240.

57. Chen JK, JR Falck, KM Reddy, J Capdevila, and RC Harris. (1998). Epoxyeicosatrienoic acids and their sulfonimide derivatives stimulate tyrosine phosphorylation and induce mitogenesis in renal epithelial cells. J Biol Chem 273:29254-29261.

58. Puri N, K Sodhi, M Haarstad, DH Kim, S Bohinc, E Foglio, G Favero, and NG Abraham. (2012). Heme induced oxidative stress attenuates sirtuin 1 and enhances adipogenesis in mesenchymal stem cells and mouse pre-adipocytes. J Cell Biochem 113:1926-1935.

59. Kershaw EE and JS Flier. (2004). Adipose tissue as an endocrine organ. J Clin Endocrinol Metab 89:2548-2556.

60. Berg AH and PE Scherer. (2005). Adipose tissue, inflammation, and cardiovascular disease. Circ Res 96:939-949.

61. Fain JN, Madan AK, Hiler ML, P Cheema, and SW Bahouth. (2004). Comparison of the release of adipokines by adipose tissue, adipose tissue matrix, and adipocytes from visceral and subcutaneous abdominal adipose tissues of obese humans. Endocrinology 145:2273-2282.

62. Kim JY, van de WE, M Laplante, A Azzara, ME Trujillo, SM Hofmann, T Schraw, JL Durand, H Li, et al. (2007). Obesity-associated improvements in metabolic profile through expansion of adipose tissue. J Clin Invest 117:2621-2637.

63. Kim DH, N Puri, K Sodhi, JR Falck, NG Abraham, J Shapiro, and ML Schwartzman. (2013). Cyclooxygenase-2 dependent metabolism of 20-HETE increases adiposity and adipocyte enlargement in mesenchymal stem cell-derived adipocytes. J Lipid Res 54:786-793.

Address correspondence to: Dr. Nader G. Abraham Departments of Medicine and Pharmacology New York Medical College 15 Dana Road Basic Science Building, Room 509 Valhalla, NY 10595-1554

E-mail: nader_abraham@nymc.edu

Received for publication March 18, 2016

Accepted after revision May 20, 2016

Prepublished on Liebert Instant Online May 25, 2016 